\title{
Extraction Techniques for Generic Cascaded Synthesis
}

\author{
Fabien Seyfert \\ FACTAS \\ INRIA \\ Sophia-Antipolis, France \\ fabien.seyfert@inria.fr
}

\author{
$\mathrm{Ke}-\mathrm{Li} \mathrm{Wu}$ \\ dept. of Electronic Engineering \\ The Chinese University of Hong Kong \\ Hong Kong \\ klwu@ee.cuhk.edu.hk
}

\author{
Smain Amari \\ The Royal Military College of Canada \\ Kingston, Canada \\ smain.amari@rmc.ca
}

\author{
Yan Zhang \\ dept. of Electronic Engineering \\ The Chinese University of Hong Kong \\ Hong Kong \\ yzhang@link.cuhk.edu.hk
}

\begin{abstract}
The aim of this paper is to demonstrate that extraction techniques can be efficiently combined with coupling matrix synthesis techniques. An extension of a recently presented method based on a functional decomposition of the scattering matrix is introduced in order to add dangling resonators based on non resonating nodes among possible buildings blocks. This leads to a complete analytical procedure for hybrid realisations including classical $\mathrm{N}$-tuplets, frequency depending couplings, constant phase shifts and NRNs. After detailing some elementary concepts of functional extraction techniques for NRN's, a synthesis and design example is presented to illustrate the approach.

Index Terms-Coupling matrix, Dispersive couplings, Filter synthesis, Non Resonating Nodes, Transmission zeros.
\end{abstract}

\section{INTRODUCTION}

The design of modern microwave bandpass filters is most often based on normalized lowpass circuit models that contain capacitors or inductors, which represent bandpass resonators, constant or frequency-dependent coupling elements or inverters, and at times frequency-independent phase shifts, and, or non-resonating nodes (NRN's). The determination of the values of the components of the chosen model is referred to as filter synthesis. Optimization-based synthesis is the most general, and least reliable method. It requires little to no knowledge of the workings of the model and is applicable to all models but suffers from the perennial problem of convergence. Known analytical filter synthesis techniques can be divided into two major groups according to their underlying mathematical structure: a) extraction methods, and b) methods based on the transformation of the coupling matrix $(\mathrm{CM})$. One might argue that the second method is only an example of methods based on extraction followed by circuit transformations. This point is not discussed here.

The extraction method applies to cascaded structures between the input and output. It should be obvious that the success of this method depends on our ability to split the overall response of the filter into sub-responses whose "composition" reproduces the overall response. The details of the "composition" of two sub-responses depend on the nature of the sub-responses themselves. For example, in the case of the scattering matrix, the decomposition is related to the work of the mathematician Potapov [1] on the general decomposition of any loss-less S-matrix into successive chaining of elementary S-matrices. The extracted pole technique is a typical example extraction techniques [2], [3]. It can be seen as an offspring of Darlington's synthesis tailored to narrow band synthesis where ideal frequency-invariant phase shifts and inverters replace the intricate Brune, $\mathrm{C}$ and $\mathrm{D}$ sections [4]. It is important to emphasize the crucial role that the transmission zeros (TZ's) play in this method. The response of a section is determined from the TZ's it is responsible for, including those at infinity. Although filter designers are familiar with the fact that the elements of an all-pole lowpass filter are extracted at infinity, i.e. at the location of the TZ's, it is worth reiterating this fact.

An example of the methods in the second group is the coupling matrix synthesis problem [5], [6]. The mathematical foundations of this approach is the work of Kalman and Ho on the realization problem of linear dynamic systems [7]. Kalman showed not only how to determine a state-space realization of a transfer function, he also showed that all representations that give the same transfer function are equivalent up to linear state space transformations. Because of the symmetry of the coupling matrix, the class of acceptable linear statespace transformations is reduced to rotations (orthogonal transformations) of the coupling matrix with constant coupling coefficients, or more generally to congruent transformations in case frequency depending couplings are considered [8]. The important advantage of this method is that it reduces the synthesis of a coupling matrix of a given topology to a problem of linear algebra. Of course, the method assumes that a starting canonical realization is available or can be determined reliably. For example, the starting coupling matrix can be chosen as that of a folded topology which can be synthesized by extraction, or a transversal coupling matrix which can be read from partial fraction expansion of the 
admittance matrix [6]. For cases that admit a non-unique solution for a given topology, it was shown that the problem of determining all possible similarity transforms amounts to a polynomial multivariate system which can be tackled by means of Gröbner basis techniques [9] or optimisation [10]-[12]. Multiplicity is for the CM approach the enemy of simplicity. A notable exception to this is the method developed in [13] for cascaded triplets and quadruplets, where extraction is so to say carried out by means of similarity transforms based on an explicit assignment of TZ's to each n-tuplet. This technique, however, becomes increasingly complicated when considering cascaded topologies based on frequency-dependent couplings (FDC's) [14]-[16], not to mention hybrid topologies made of cascaded n-tuplets, FDC's, and non-resonating nodes. Since the increasingly stringent requirements of the communication systems of the future are very likely to be met only by hybrid filter designs involving cross coupling, phase shifts, FDC's and NRN's, there is an urgent need for more reliable synthesis techniques of this class of filters.

The first reported effort in this direction starts from the extraction of an in-line circuit with NRNs' followed by selective suppression of appropriate NRN's to generate singlets or triplets [17]. This approach was expanded further in [18] where more general blocks are obtained by means of sequential application of the star-to-mesh transformation and further similarity transformations. In [8] a method which involves two steps was presented. The first step consists in decomposing the target $S$ matrix of the filter into was a set of scattering submatrices such that $S^{1}, S^{2} \ldots S^{m}$

$$
S=S^{1} \circ S^{2} \ldots S^{m} \text {. }
$$

Here $\circ$ means that the second port of the first response is directly connected to the first port of the second one. Each of the $S^{k}$ implements a subset of the TZ's of $S$. These sub-responses $S^{k}$ are then realized by means of elementary building blocks obtained by the CM synthesis method through congruent transformations. The building blocks include $\mathrm{n}$ tpulets, inline FDC's as well as FDC based n-tuplets.

The objective of this paper is to demonstrate that dangling resonator based on NRNs or phase shifts are easily added to these collection, leading to a versatile analytical synthesis method which is able to handle all known $\mathrm{TZ}$ generating mechanisms.

\section{DANGLING RESONATOR SECTION}

\section{A. Extraction}

A typical extracted pole section is depicted in Fig. 1. It is meant for extraction of a TZ at $z_{0}=-j b / L$. Using the notations of [8] and in matrix form the S matrix $S^{E P}$ of the extracted pole section is:

$$
\begin{aligned}
S^{E P} & =\left[\begin{array}{cc}
0 & e^{j \phi_{1}} \\
e^{j \phi_{1}} & 0
\end{array}\right] \circ \\
& \frac{1}{s-z_{0}-1 / \zeta_{0}}\left[\begin{array}{cc}
-\gamma / \zeta_{0} & s-z_{0} \\
s-z_{0} & -1 /\left(\gamma \zeta_{0}\right)
\end{array}\right] \circ\left[\begin{array}{cc}
0 & e^{j \phi_{2}} \\
e^{j \phi_{2}} & 0
\end{array}\right]
\end{aligned}
$$

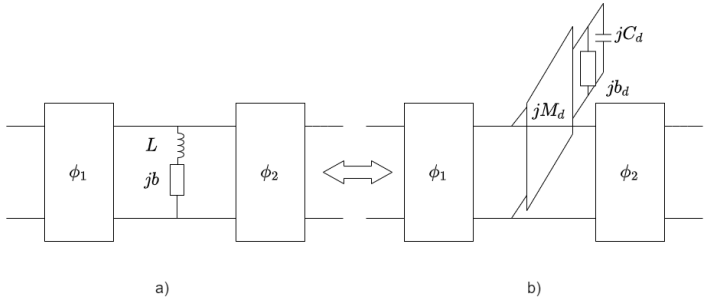

Fig. 1. Extracted pole section as: a) a shunt bandstop resonator b) dangling bandpass resonator

where $\gamma=-1$ and $\zeta_{0}=-1 /(2 L)$. Suppose now that a purely imaginary $\mathrm{TZ} z_{0}$ is to be extracted by such a section from a $2 \times 2$ loss-less, reciprocal scattering matrix $G$. The first ideal phase shift, call its matrix $G^{\phi_{1}}$, is extracted in order to yield a remainder matrix $G^{1}$ such that,

$$
G=G^{\phi_{1}} \circ G^{1},
$$

and $G_{11}^{1}\left(z_{0}\right)=-1$ holds. This leads to

$$
\phi_{1}=\left(\operatorname{Arg}\left(G_{11}\left(z_{0}\right)\right)-\pi\right) / 2 .
$$

In a second step the shunt bandstop resonator section, call its scattering matrix $G^{\zeta_{0}}$, is classically extracted by matching angular derivative values, that is $\zeta_{0}=\operatorname{Ang}\left(G_{11}^{1}\right)\left[z_{0}\right]$. At this point the inductor $L$ and susceptance $j b$ are entirely determined as,

$$
L=-1 /\left(2 \operatorname{Ang}\left(G_{11}^{1}\right)\left[z_{0}\right]\right), \quad b=-L z_{0} / j .
$$

Eventually the last phase shift, call its scattering matrix $G^{\phi_{2}}$, is extracted depending on the phase condition that is desired for the remainder response $G^{3}$. More precisely we have,

$$
G=G^{\phi_{1}} \circ G^{\zeta_{0}} \circ G^{2}=G^{\phi_{1}} \circ G^{\zeta_{0}} \circ G^{\phi_{2}} \circ G^{3} .
$$

If a classical n-tuplet section (with or without FDC's) is to be extracted from $G^{3}$ implementing at least one TZ at infinity then $\phi_{2}$ is chosen in order to have $G_{11}^{3}(\infty)=1$. This is ensured by

$$
\phi_{2}=\operatorname{Arg}\left(G_{11}^{2}(\infty)\right) / 2 .
$$

In case another $\mathrm{TZ} z_{2}$ is to be extracted by a dangling resonator, $\phi_{2}$ corresponds to to the angle $\phi_{1}$ and is chosen so as to ensure $G_{11}^{3}\left(z_{2}\right)=-1$ : this corresponds to the usual iteration scheme of extracted poles synthesis.

\section{B. Outline of the synthesis method}

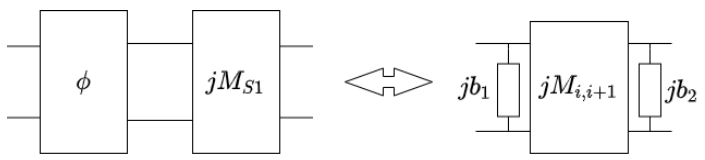

Fig. 2. Equivalence between frequency invariant $2 \times 2$ blocks

As in [8] the synthesis method starts with a $2 \times 2$ response $S$ to be realised. According to the cascaded circuital blocks that are intended for its realisation and the TZ partition it induces, $S$ is decomposed as in (1). The particularity here 
is that some of sub-blocks may be composed of dangling resonators. The sub-blocks corresponding to n-tuplets are realized as circuits which are eventually connected together. At the interconnection of an extracted pole or NRN section and a n-tuplet, a phase shift is cascaded with an inverter: this frequency independent admittance is replaced by its equivalent circuit as depicted on Fig. 2, that is an inverter surrounded by two susceptances. By this procedure all phase shifts are replaced by their inverters based equivalent and a cascaded coupled resonator realisation is obtained containing possibly n-tuplets, FDCs and NRN based dangling resonators.

\section{SYNTHESIS AND DESIGN EXAMPLE}

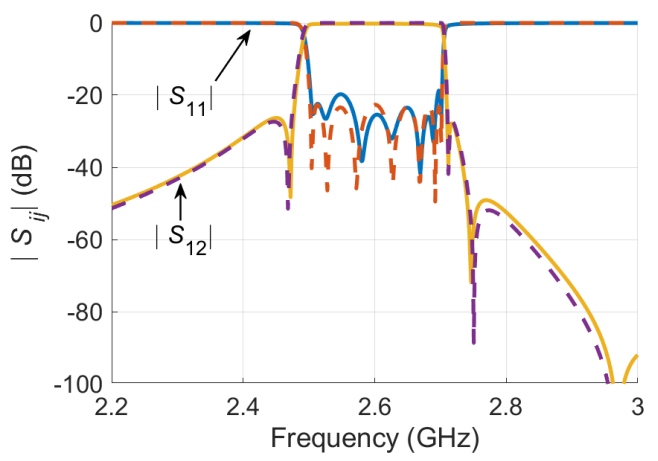

Fig. 3. The synthesized 7-4 assymetric response (dashed lines) and EM simulated response (solid lines).

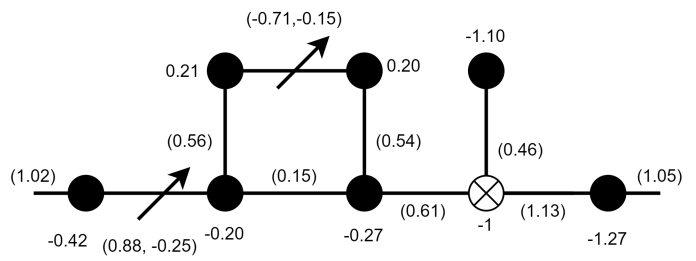

Fig. 4. Hybrid topology composed of the cascade of a FDC duplet with a FDC based quadruplet, a NRN based dangling resonator and a usual bandpass resonator. Coupling values are noted within braces: the second value, when noted is the coupling slope. All values are meant for the normalized domain.

The synthesis and design of a $7^{\text {th }}$ order response (see dashed lines in Fig. 3) with 4 normalized TZs at $-1.35 j, 1.1 j, 1.45 j$, and $3.45 j$, is considered according to the hybrid topology of Fig. 4. The filter centers at $f_{0}=2.6 \mathrm{GHz}$ with a bandwidth $B W=0.2 \mathrm{GHz}$. The in-band return loss of the synthesized response is $23 \mathrm{~dB}$. The realisation of the $\mathrm{TZ}$ at $3.45 j$ is considered with the dispersive duplet, while the dispersive quadruplet takes care of the TZ's at $-1.35 j$ and $1.45 j$. Finally the $\mathrm{TZ}$ at $1.1 j$ is realized by means of dangling resonator at the output. According to this TZ distribution the response $S$ is split in three sub-responses $S=S^{1} \circ S^{2} \circ S^{3}$ where the penultimate corresponds to the dangling resonator section described previously. Coupling values obtained by realizing each circuit block independently and connecting them together are shown in Fig. 4. We made use of the re-scaling feature inherent to NRN's [3] in order to obtain acceptable couplings values for the frequency offset of the NRN and its couplings to resonators.

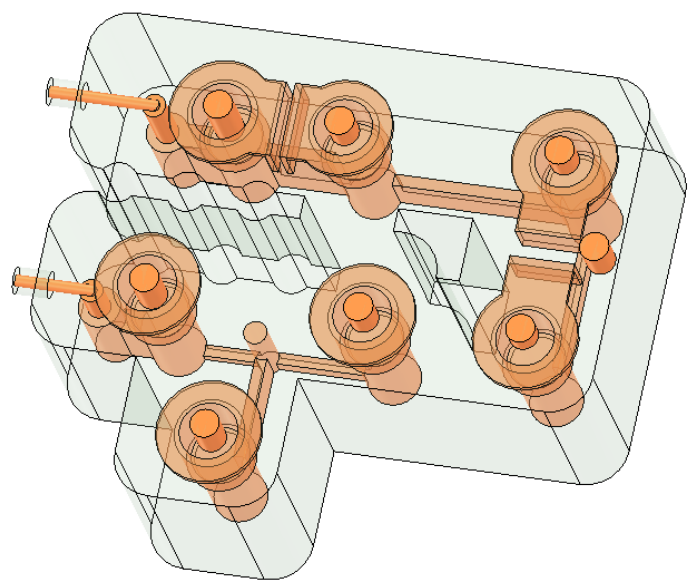

Fig. 5. Realization of the filter.

The EM model of the filter is depicted in Fig. 5, where a stub with one end short and one end open is utilized to realize the NRN. It's connected to the three adjacent resonators by ridges, the height of which controls the positive coupling values similar to the ridges between resonators. The dispersive coupling structure is realized with a ridge connecting two resonators and two bended plates on top them [8]. By properly adjusting the height of the ridge and the width of the gap between the two parallel plates, the desired dispersive coupling slope and its constant part can be realized. Eventually, the simulated response of the EM model is superimposed in Fig. 3 on the synthesized response, demonstrating a good agreement of theory and practical implementation.

\section{REFERENCES}

[1] V. Potapov, "The multiplicative structure of $j$-contractive matrix functions," American mathematical society translations, vol. 15, pp. 131243, 1960.

[2] J. Rhodes and R. Cameron, "General extracted pole synthesis technique with applications to low-loss te/sub011/ mode filters," IEEE Transactions on Microwave Theory and Techniques, vol. 28, no. 9, pp. 1018-1028, 1980.

[3] S. Amari and G. Macchiarella, "Synthesis of inline filters with arbitrarily placed attenuation poles by using nonresonating nodes," IEEE Transactions on Microwave Theory and Techniques, vol. 53, no. 10, pp. 3075-3081, 2005.

[4] H. J. Carlin and P. P. Civalleri, Wideband Circuit Design, 1st ed. NY, USA: CRC Press, 1998.

[5] R. J. Cameron, A. R. Harish, and C. J. Radcliffe, "Synthesis of advanced microwave filters without diagonal cross-couplings," IEEE Transactions on Microwave Theory and Techniques, vol. 50, no. 12, pp. 2862-2872, Dec 2002

[6] R. J. Cameron, C. M. Kudsia, and R. Mansour, Microwave Filters for Communication Systems: Fundamentals Design and Applications, 2nd ed. Hoboken, NJ, USA: Wiley, 2018.

[7] T. Kailath, Linear Systems, ser. Information and System Sciences Series. Prentice-Hall, 1980.

[8] Y. Zhang, F. Seyfert, S. Amari, M. Olivi, and K.-L. Wu, "General synthesis method for dispersively coupled resonator filters with cascaded topologies," IEEE Transactions on Microwave Theory and Techniques, vol. 69, no. 2, pp. 1378-1393, 2021 
[9] R. J. Cameron, J.-C. Faugere, F. Rouillier, and F. Seyfert, "Exhaustive approach to the coupling matrix synthesis problem and application to the design of high degree asymmetric filters," International Journal of $R F$ and Microwave Computer-Aided Engineering, vol. 17, no. 1, pp. 4-12, 2006.

[10] S. Amari, "Synthesis of cross-coupled resonator filters using an analytical gradient-based optimization technique," IEEE Transactions on Microwave Theory and Techniques, vol. 48, no. 9, pp. 1559-1564, Sept 2000.

[11] L. Szydlowski, A. Lamecki, and M. Mrozowski, "Coupled-resonator filters with frequency-dependent couplings: Coupling matrix synthesis," IEEE Microw. Wireless Compon. Lett., vol. 22, no. 6, pp. 312-314, 2012.

[12] L. Szydlowski, N. Leszczynska, and M. Mrozowski, "Dimensional synthesis of coupled-resonator pseudoelliptic microwave bandpass filters with constant and dispersive couplings," IEEE Trans. Microw. Theory Tech., vol. 62, no. 8, pp. 1634-1646, 2014.

[13] S. Tamiazzo and G. Macchiarella, "An analytical technique for the synthesis of cascaded n-tuplets cross-coupled resonators microwave filters using matrix rotations," IEEE Trans. Microw. Theory Tech., vol. 53, no. 5, pp. 1693-1698, 2005.

[14] — "Synthesis of cross-coupled filters with frequency-dependent couplings," IEEE Trans. Microw. Theory Tech., vol. 65, no. 3, pp. 775782, 2017.

[15] Y. He, G. Macchiarella, G. Wang, W. Wu, L. Sun, L. Wang, and R. Zhang, "A direct matrix synthesis for in-line filters with transmission zeros generated by frequency-variant couplings," IEEE Trans. Microw. Theory Tech., vol. 66, no. 4, pp. 1780-1789, 2018.

[16] Y. He, G. Macchiarella, Z. Ma, L. Sun, and N. Yoshikawa, "Advanced direct synthesis approach for high selectivity in-line topology filters comprising $n-1$ adjacent frequency-variant couplings," IEEE Access, vol. 7, pp. 41 659-41668, 2019.

[17] S. Amari, "Direct synthesis of cascaded singlets and triplets by nonresonating node suppression," in 2006 IEEE MTT-S International Microwave Symposium Digest, 2006, pp. 123-126.

[18] S. C. Mejillones, M. Oldoni, S. Moscato, G. Macchiarella, M. D'Amico, G. G. Gentili, and G. Biscevic, "Unified analytical synthesis of cascaded n-tuplets filters including nonresonant nodes," IEEE Transactions on Microwave Theory and Techniques, pp. 1-1, 2021. 